\title{
QR Code Based Patient Medical Health Records Transmission: Zimbabwean Case
}

\author{
Sibusisiwe Dube, Siqabukile Ndlovu, Thambo Nyathi, \\ and Khulekani Sibanda \\ National University of Science and Technology, \\ Bulawayo, Bulawayo Province, Zimbabwe
}

\author{
sibusisiwe.dube@nust.ac.zw, siqabukile.sihwa@nust.ac.zw, \\ thambo.nyathi@nust.ac.zw, khulekani.sibanda@nust.ac.zw
}

\begin{abstract}
In Zimbabwe the health care delivery system is hierarchical and patient transfer from the lower level to the next higher level health care facility involves patients carrying their physical medical record card. A medical record card holds information pertaining to the patient's medical history, pre-existing allergies, medical health conditions, prescribed medication the patient is currently taking among other details. Recording such patient information on a medical health card renders it susceptible to tempering, loss, and misinterpretation as well as susceptible to breaches in confidentiality. In this paper, we propose the application of Quick Response (QR) codes to secure and transmit this sensitive patient information from one level of the health care delivery system to another. Other security methods such as steganography could be used, but in this paper we propose the use of QR codes owing to the high proliferation of mobile phones in the country, high storage capacity, flexibility, ease of use and their capability to maintain data integrity as well as storage of data in any format.
\end{abstract}

Keywords QR code(s), medical health records, mobile phone, patient, medical health card

\section{Introduction}

The health care delivery system in Zimbabwe has a hierarchical structure depicted in Figure 1.

In an ideal situation the community, this could be a village in a rural setting or a locale in an urban setting is manned by an individual with basic medical training referred to as a community health worker (CHW)(Ministry of Health \&Child Welfare Zimbabwe, 2013). The CHW is not usually attached to any physical structure such as a clinic but is mobile within the community

Material published as part of this publication, either on-line or in print, is copyrighted by the Informing Science Institute. Permission to make digital or paper copy of part or all of these works for personal or classroom use is granted without fee provided that the copies are not made or distributed for profit or commercial advantage AND that copies 1) bear this notice in full and 2) give the full citation on the first page. It is permissible to abstract these works so long as credit is given. To copy in all other cases or to republish or to post on a server or to redistribute to lists requires specific permission and payment of a fee. Contact Publisher@InformingScience.org to request redistribution permission. he/she can make home visits and be visited to attend to minor ailments. This scenario is no longer the case in urban settings as people are not restricted in choice when it comes to health issues, but is still more prevalent in rural areas which house the majority of the Zimbabwean populace. If a patient who is attended to by the CHW requires further treatment the $\mathrm{CHW}$ verbally refers them to the community health clinic/facility 
(CHF). The CHF is manned by nurses and has a periodical visiting doctor although the ideal situation will be to a have one stationed there permanently. In both instances i.e. successful treatment or referral there is currently no record kept by the CHW. This is an inconvenience as the patient will have to re-narrate their health problem when they are referred to the clinic. At the CHF, the nurse will perform the standard basic medical examination procedures which may include but are not limited to checking the weight, blood pressure and temperature of the patient. These details, together with the narrated health problem are recorded in a medical record card. At this point of the hierarchy the nurse repeats this process for every new patient. The nurse, at the CHF, has the authority to diagnose and treat except if the problem is major and requires the attention of a visiting doctor. Upon successful diagnosis, the patient either gets free medication or gets a prescription to purchase the medication and returns home without giving feed back to the CHW. If diagnosis and/or treatment are not successful the patient is further transferred to a referral district hospital (DH) which is at the next level in the hierarchy.

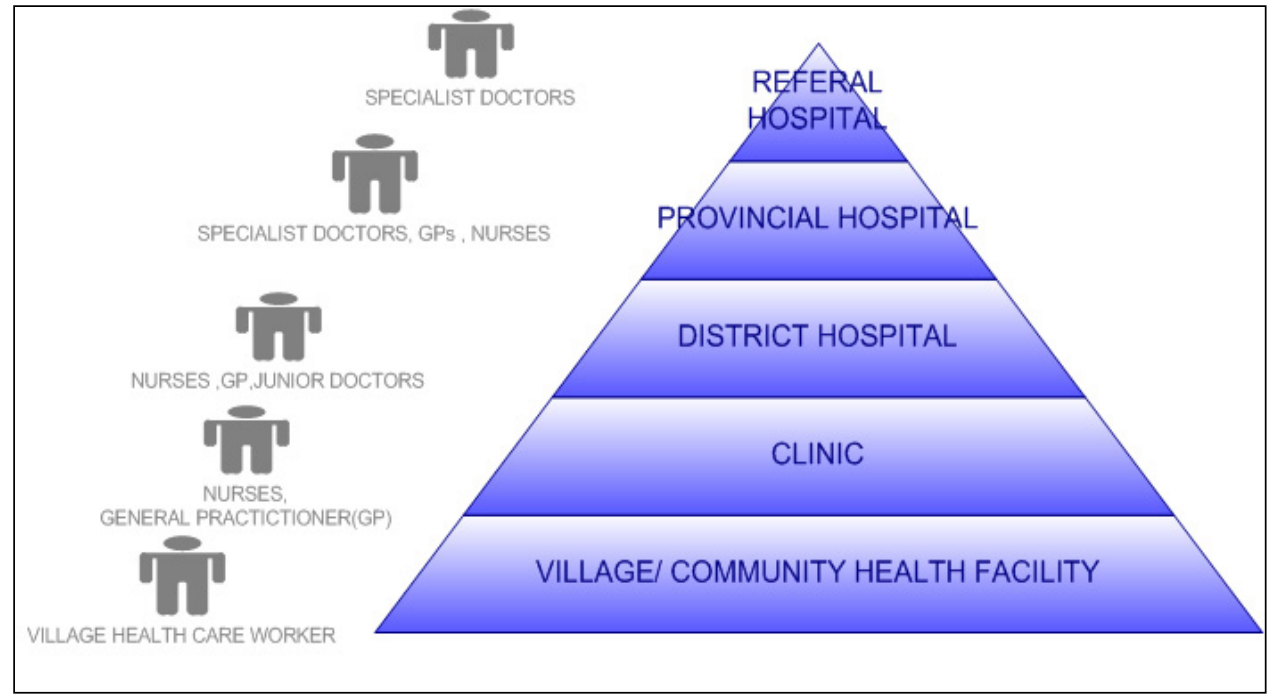

Figure. 1 Structure of the Health System (Ministry of Health and Child Welfare Zimbabwe, 2013)

On referral, the patient maintains the same medical record card with the details of their history, diagnosis, condition and comments from either the clinic nurse or the clinic visiting doctor. The same medical record card is maintained possibly to the highest level in the hierarchy indicating what was observed and prescribed all the way from the clinic to the referral hospital where the patiently is possibly treated successfully. It is maintained throughout the monitoring and review process downward from the referral hospital to the clinic as indicated in Figure 2. 


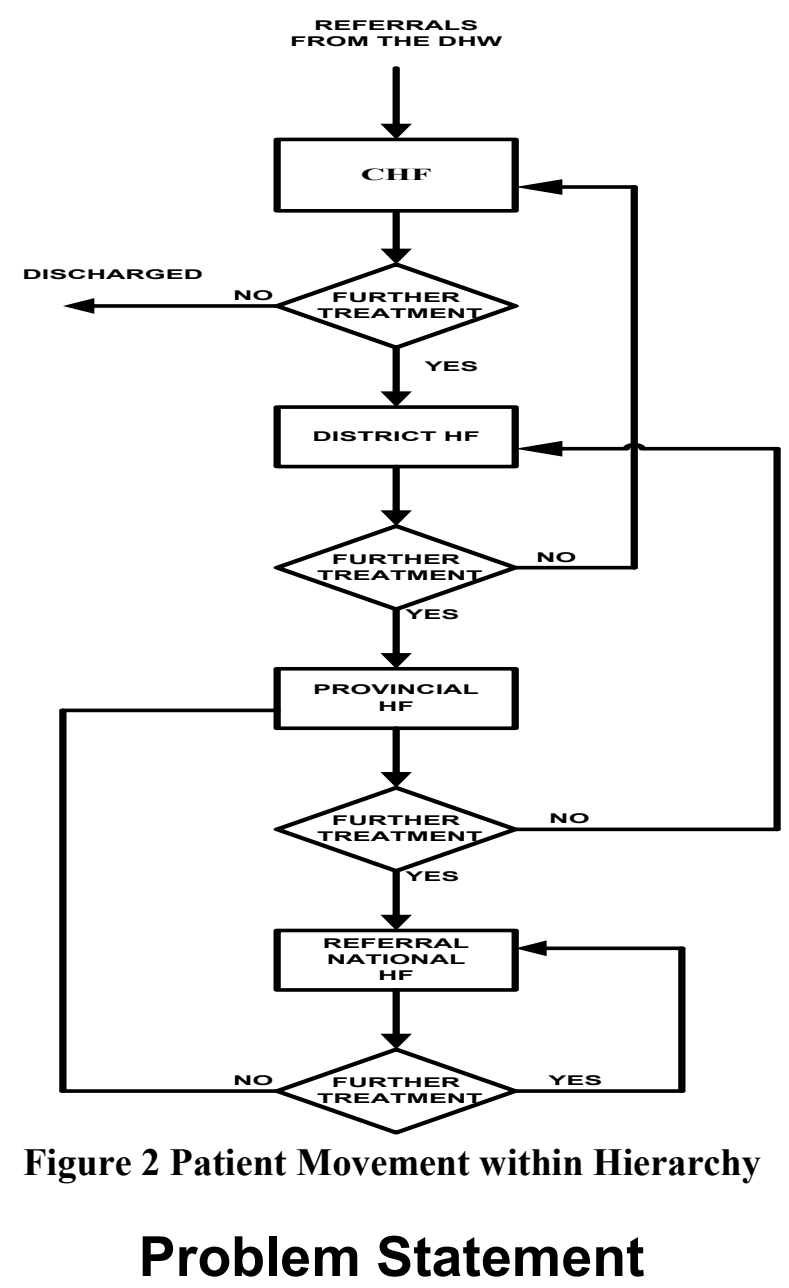

During the process of patient transfer the patient's medical information is vulnerable and susceptible to various degrees of integrity compromise. For example the medical record card may get lost hence losing all the important patient medical information. It is also susceptible to information tempering either by the patient or other third parties. For example, the medical record card can be scribbled on, soiled, illegally altered or torn. An additional problem is that the medical record card may be accessible to unauthorised individuals who may eavesdrop the information on the card and maliciously release it hence violating the privacy of the patient. Most importantly, despite access by unintended recipients, the details on the medical record card are also prone to misinterpretation owing to illegible handwriting. All these problems may result in a breached security based on the lack of confidentiality, integrity and availability of the authentic patient medical information. The research thus seeks to find an answer to the question as to how the use of QR codes can enable secure delivery of patient medical information.

\section{Aim and Objectives}

The aim of the paper is to evaluate the capability of the QR code to secure patient medical information during transit maintaining confidentiality, integrity and availability at the intended destination within the hierarchical structure of the health delivery system in Zimbabwe. We also advocate a secure and affordable information transmission method that ensures the delivery of complete, accurate and well documented patient medical records. 


\section{Related Work}

\section{Method for Privacy-Protecting Display and Exchange of Emergency Information on Mobile Devices}

Salvador and Christian (2012) developed a solution that allows users to decode patient information and automatically notify authorized contacts of the nature of the emergency and the location where the patient is being cared for. A QR code is created by encoding the mobile phone user's emergency and medical information (such as the name and contact details of the user's next of kin, their doctor or specialist, blood group, allergies, etc.). The created code image is then used as a wallpaper on the phone. This allows anyone to scan the code without unlocking the phone. Scanning and decoding the QR code can be accomplished with any standard QR Code reader, but when read with QRCScan, additional information is retrieved and revealed. This helps emergency medical responders by enabling them to provide medical care with a more informed base.

\section{QR Codes Could Minimize Medical Errors Made by First Responders}

Drug mistakes are the most common recorded errors in the healthcare industry all over the world (Dong-Hee, Jaemin, \& Byeng-Hee, 2012). According to the same authors, medication errors affect more than 1.5 million people in US each year which translates to $\$ 3.5$ billion spent on related treatments. QR codes could minimise the chances of these errors by offering accurate information to first responders who can easily read this information by using a QR code scanner on their Smartphones. In this research, QR codes holding the patient's information are tattooed onto an area easily reachable by first responders. The information would also provide accountability when a treatment does not go the way it was planned. Many of these medical errors are preventable through an increase in communication between healthcare providers, improved patient identification, and consumer knowledge (Dong-Hee, Jaemin, \& Byeng-Hee, 2012).

\section{A Novel Secret sharing technique using QR Code}

In this paper, (Jun-Chou, Yu-Chen, \& Hsien-Ju, 2013) propose a secret sharing mechanism to enhance the security and data privacy for QR codes. The secret sharing scheme was first proposed by Shamir in 1979 (Shamir, 1979). The main idea of the secret sharing scheme divides a secret into $\mathrm{n}$ shadows or called shares. Anyone cannot decrypt the original secret from their own share. The secret can be recovered only when any of $t$ out of $n$ shadows $(t<=n)$ are held together. The proposed technique improves data security during data transmission. On the other hand, the proposed technique does not need to establish a back-end database beforehand for contents searching. It direct embeds the secret data into tags therefore the proposed technique can save a lot of hardware cost and software maintenance. The proposed technique can be applied to some applications such as electronic tickets, airline luggage inspection, medical e-health system, and other fields. However, the major drawback is that the recovery process requires all the stakeholders to come together which may not always be possible in the Zimbabwean rural setting. This would also affect the speed of getting the information and would mean the patient would have to wait for their information to be decoded.

\section{Proposed Solution}

$\mathrm{QR}$ codes can be implemented to solve the problems stated in the problem statement section of this paper. QR codes are 2 dimensional barcodes which provide instant access to information (Espejel-Trujillo, Castillo-Camacho, Nakano-Miyatake, \& Perez-Meana, 2012), (Gao \& Prakash, 2007). They allow for storage of higher amounts of data compared to conventional barcodes 
which is appropriate for the proposed solution. This data can be in the form of numbers (maximum 7,089 characters), text (maximum 4,296 characters), binary (maximum 2,953 bytes) or kanji characters (maximum 1,817 characters) and this would allow even pictures to be sent. An interesting feature of QR codes is their capability to perform error correction in case the parts of the code are damaged (Espejel-Trujillo, Castillo-Camacho, Nakano-Miyatake, \& Perez-Meana, 2012). This means even if the code is damaged, the information in it will still be valid and usable. They can be coded and decoded on smartphones through the in-built cameras as long as the smartphone is equipped with a QR code reader.

QR code readers can be downloaded for free from the internet which another advantage of the proposed solution is given that Zimbabwe is a developing country. QR codes can handle information in a variety of formats such as URLs, contact information, SMS and plain text among others (Law \& So, 2010). As research has shown, QR codes have proved to be useful in several fields. In the medical field, they have been used on food and drink packages to show the nutritional information these products carry (Yeh, You, \& Jong, 2008), as assistive tools for emergency workers and as transaction authentication tools for medical billing (Starnberger, Froihofer, \& Goeschka, 2009.). QR codes have been and can be tattooed onto patients (Dong-Hee, Jaemin, \& Byeng-Hee, 2012) and this helps particularly in situations where patients are in a serious condition.

The tattooed information can still be decoded as and when needed as it has a direct link with the specific patient's medical health records. Patients can also decode QR coded instructions on how their medication should be administered. However due to cultural constraints, this paper recommends that the patient medical health information, prescriptions and the instructions for the medicine administration be restricted to the QR codes encoded and decoded via the smartphones and not tattooed. This means at the source, the patient medical health details are encoded and at the destination decoded using a smartphone.

\section{Methodology}

The research approach followed is the Action Research methodology. In Action Research the researcher tries to provide a service to a research "client", often an entity, and at the same time add to the body of knowledge in a particular domain (Nyathi, Dube, Sibanda, \& Mutunhu, 2013). For example, in a technology-related domain, an Action Research study could involve the researcher introducing a new technology, and at the same time studying the effects of the technology in that entity. The methodology best suits the problem at hand as it involves the participation of the subjects in the provision of a solution to the security problem. Action research is used in real life situations, rather than in artificial, experimental studies, as its primary focus is on solving real life problems. It can be used by social scientists for preliminary or pilot research. It is mostly used when the situation is too ambiguous to come up with a precise research question. Mostly it is chosen when circumstances require flexibility, the participation of the people in the research, or when change must take place quickly or fully. Figure 3 depicts the phases in an Action Research methodology.

\section{Stage 1: Diagnosis}

The diagnosis stage involves the identification of an enhancement opportunity or a general problem to be solved at the client entity. An enhancement opportunity has been identified in the transfer of patient information from one level to the other. Since patient information is transmitted on a medical record card it is prone to loss, misinterpretation, eavesdropping and unauthorized editing hence the need for a more secure method of transmission capable of countering such identified problems. QR codes can be used to securely transmit confidential patient information. Data transmission using QR codes is reliable as they can still be read even when they are damaged. 


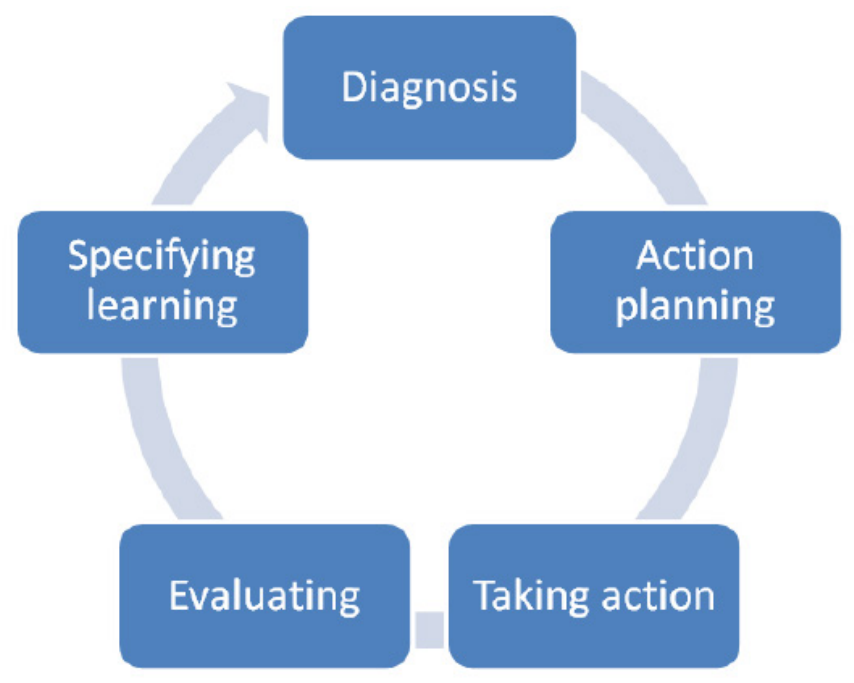

Figure 3: Stages of Action Research Methodology

\section{Stage 2: Action Planning}

Action planning involves considering the alternative solutions to attain the enhancement or solve the identified problem(s). The possible solutions include the design, development and: implementation of a centralized database to store patient information; which can be transmitted via the email, which can further be secured by steganography. However there is a challenge of infrastructure particularly in the lower levels of the hierarchy of the health delivery system. These areas do not have electrical power to ensure the use of computing technology. This renders this alternative not feasible. The other alternative is the use of an Android app (Reiser \& Bruce, 2008.) to transfer information between stations. This alternative is also not feasible given that the available apps are not customized for the given situation. The use of QR codes as both the storage and transmission method of medical records is the most feasibly solution to the problem. This is a multipurpose method which comes in handy in the given economic environment as information can be recorded, stored, transmitted and retrieved using a single device.

\section{Stage 3: Action Taking}

In the action taking stage the best course of action among those considered in the previous stage is selected and implemented. The alternative selected to implement a solution to the problem is the use of QR codes. There are a number of reasons why this option was selected. The QR code technology is easy to deploy, fast and economical. QR codes allow their contents to be decoded at high speed (Espejel-Trujillo, Castillo-Camacho, Nakano-Miyatake, \& Perez-Meana, 2012), (Rouillard, 2008). They carry information in two directions i.e. vertically and horizontally, hence they are capable of handling large amounts of data. QR codes can be combined into one large symbol. One symbol can then be divided into 16 separate symbols making the data capacity extremely large. The other alternatives considered in the Action Planning phase were not selected because of the following disadvantages: performance degradation as the size of the database increases, issues of scalability since data for the whole nation has to be centrally stored, inaccessibility when offline, security vulnerabilities, dependence on electrical power, resource intensive as each point will have to be equipped with at least the basic computing infrastructure which includes hardware, software network connection and communication devices and all these have a high initial and maintenance costs. 
On the other hand the use of QR codes is more feasible due to accessibility to low cost smartphones and the freely available QR code application which can be downloaded from the internet at no cost except the insignificant internet connectivity cost. The implementation of the proposed system will entail a patient visiting a low level health care institution were a caregiver diagnoses and treats them. The diagnosis and treatment are recorded on a medical record card that should be safely kept at this health facility for future reference instead of the patient carrying it around. If treatment fails at that level, the caregiver generates a QR code from the recorded information and encrypts it before sending it to the next higher level health care facility. When the patient is referred, they will only need to carry their national identity card so that their data can be retrieved.

QRDroid is an Android application used to generate and decode QR codes. It is freely available for downloading on the internet. The application has two modules: a code generator and a code reader (Kheder \& Alvi, 2013). The QR code generator module is used to generate the QR code where the user selects the "Plain Text" option on the type of QR code to generate and then types the medical information of the patient in the console. Before sending, the created code is encrypted for extra security. The benefit of this application is that it allows for the generation of QR codes using any other type of information like pictures. This allows x-rays, scans and the actual image of the patient's initial health status when they first visited the lower health care facility to be encoded and transmitted for further examination. The generated QR code can then be sent to the next higher level health care institution were the patient has been referred to as an MMS or any communications application that can send and receive any type of information like Whatsapp. At the receiving end the code is scanned using the reading module and the decryption key is entered to decode information.

\section{Stage 4: Evaluating}

This stage involves the study of the outcomes of the selected course of action. Using QR codes has proved to be a very secure and affordable method of patient medical health information transmission. The information is delivered to the intended destination in its expected and intended form. The patient information integrity and confidentiality are maintained and this information is available as and when required. It neither disappears, leaks nor allows modification while on transit. It also retains its integrity as it does not suffer from misinterpretation caused by illegible handwriting. As long as the information is sent to the intended recipient, QR codes offer confidentiality of patient information.

\section{Stage 5: Specifying Learning}

The specifying learning stage reviews the outcomes of the evaluating stage and builds knowledge in the form of a model describing the situation under study. The major challenge faced in Zimbabwe is a lack of a centralized system that keeps medical information of all patients who visit public hospitals. The system we have proposed helps when it comes to transferring patient information from one level to another level in the health care delivery system but it was going to be of more help if that information was stored centrally and any changes in a patient's health card to reflect in the central system.

\section{Discussion and Conclusion}

QR codes are a very reliable means of transmitting patient information within the health care delivery system hierarchy. The QR codes ensure confidentiality, integrity and availability of the transmitted patient information from one level of the health delivery system hierarchy to the other. This is essential for preventing high mortality rates possibly caused by wrong diagnosis and treatment as a result of lost or misinterpreted records. Figure 4 is an extract of an actual child 
health card used in Zimbabwe showing the type information that is recorded in it. Looking at the figure immediately brings out the issue of illegible handwriting.

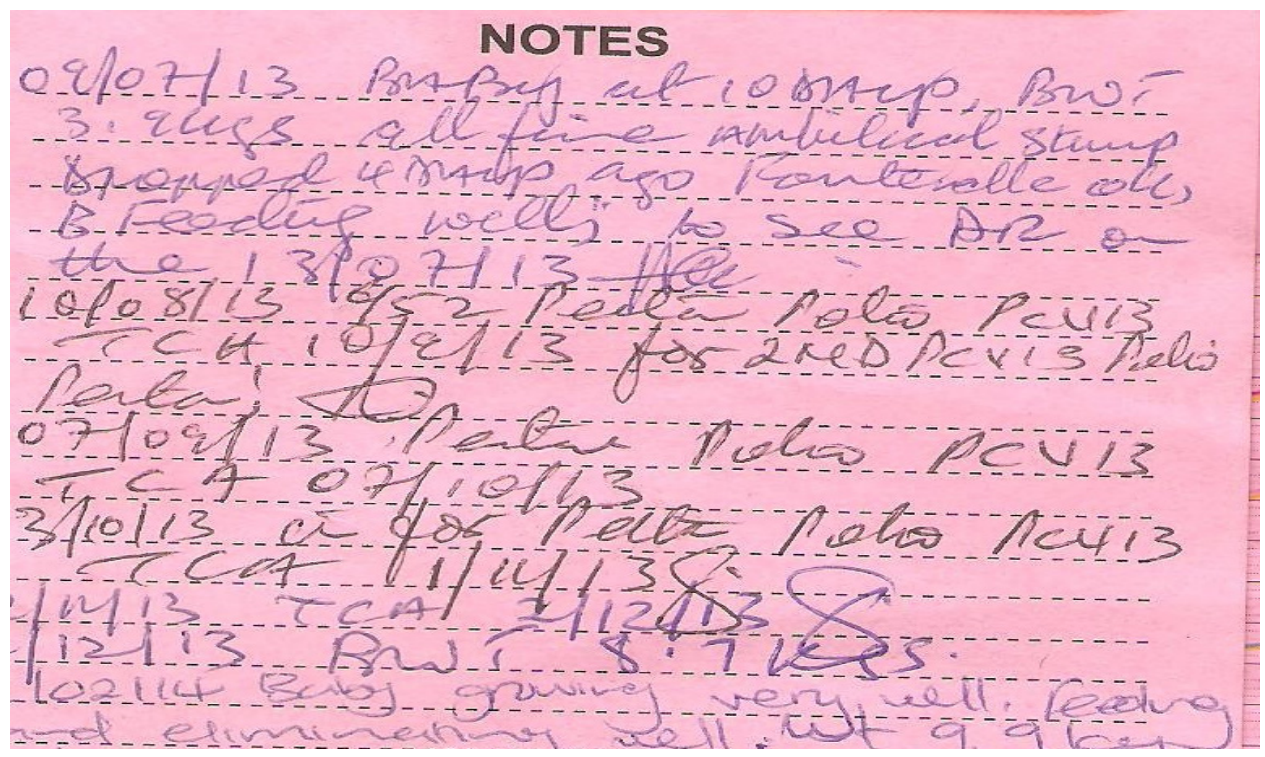

Figure 4: An extract of a QR coded baby health card.

Figure 5 is a QR code produced from the information recorded in the child health card in Figure 3. This QR code can then be encrypted using the encryption function found in QRDroid and passed on to another health centre or doctor in case of referral. This is proof that the use of QR codes is a highly secure, reliable and trustworthy method to transfer sensitive data.

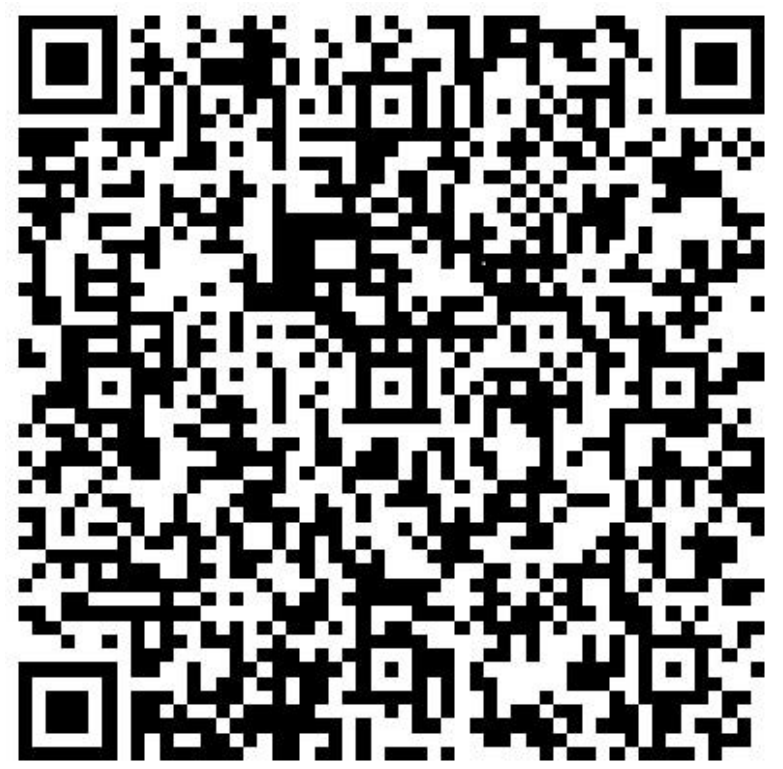

Figure 5: QR code containing information on the baby health card

System deployment expense, user education and IT literacy are parameters perceived to hamper the adoption process. However as already indicated the critical infrastructure for system adoption already exists and Zimbabwe enjoys the highest literacy rate in Africa of $91 \%$ which is likely to make IT literacy conversion seamless. 


\section{References}

Dong-Hee, S., Jaemin, J., \& Byeng-Hee, C. (2012). The psychology behind QR codes: User experience perspective. Computers in Human Behavior 28 , 1417-1426.

Espejel-Trujillo, A., Castillo-Camacho, I., Nakano-Miyatake, M., \& Perez-Meana, H. (2012). Identity Document Authentication Based on VSS and QR Codes. Procedia Technology 3, 241 - 250.

Gao, J., \& Prakash, L. J. (2007). Understanding 2D-barcode technology and applications in m-commerce design and implementation of a 2D barcode processing solution". Proceedings of the Computer Software and Applications Conference,. Bejing,.

Jun-Chou, C., Yu-Chen, H., \& Hsien-Ju, K. (2013). A Novel Secret Sharing Technique Using QR Code. International Journal of Image Processing (IJIP), Volume (4) : Issue (5).

Kheder, L. S., \& Alvi, A. (2013). Authentication System Using Quick Response Code. International Journal of Management, IT and Engineering, Vol 3, Issue 2, 373-38.

Law, C., \& So, S. (2010). , QR codes in education. Journal of Educational Technology. Development and Exchange, 3(1), 85-100.

Ministry of Health and Child Welfare Zimbabwe, A. (2013, October 20). Hierarchical structure of the health delivery system. Retrieved from mohcc.gov.zw: http://www.mohcc.gov.zw/

Nyathi, T., Dube, S., Sibanda, K., \& Mutunhu, B. (2013). Poultry Contractual Farming Decision Support System. IST Africa 2013 Proceedings (pp. 1-8). Kenya: IST Africa.

Reiser, S., \& Bruce, R. (2008.). Service learning meets mobile computing. Proceedings of the Annual Southeast Regional Conference. Auburn, Alabama.

Rouillard, J. (2008). Contextual QR codes. Proceedings of the 3rd International Multi-Conference on Computing in the Global Information Technology, . Athens, Greece.

Salvador, A., \& Christian, P. (2012). Method for Privacy-Protecting Display and Exchange of Emergency Information on Mobile Devices.

Shamir, A. (1979). "How to Share a Secret. Communication of the ACM, 22(11), 612-613.

Starnberger, G., Froihofer, L., \& Goeschka, K. ( 2009.). QR-TAN: Secure mobile transaction authentication. Proceedings of the Availability, Reliability and Security, . Fukuoka, Japan.

Yeh, Y., You, J., \& Jong, G. (2008). The 2D bar-code technology applications in medical information management. Proceedings of the Intelligent Systems Design and Applications. Kaohsiung, Taiwan,.

\section{Biographies}

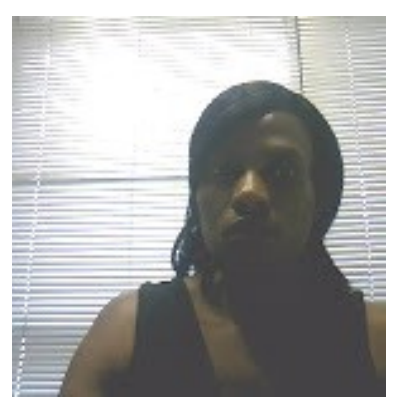

Sibusisiwe Dube is a $\mathrm{PhD}$ in Information Systems student at the University of Cape Town, South Africa. She has worked as a Lecturer and her research interest is on technology enabled education. The research work is available in the IEEE sponsored conference proceedings such as IST as well as other conference proceedings such as the e-skills, ECEL, ICAT and SACLA. Mrs Dube holds an MSc in Computer Science, and BSc in Information Systems. 


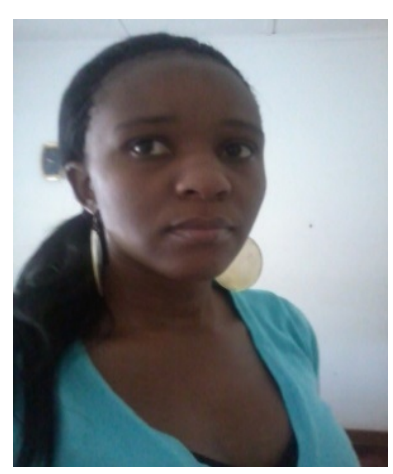

Siqabukile Ndlovu is a Computer Science Maters' graduate who has been teaching at the National University of Science and Technology (Zimbabwe) for the past five years. Her publishing career started in 2013 and to date, she has collaboratively published two articles with international journals ("Beacon Frame Manipulation to Mitigate Rogue Access Points: Case of Smartphone Rogue Access Points.": International Journal of Advanced Computer Technology (COMPUSOFT). 02/2014; Three(Two):576 and "Optimisation of the Linear Probability Model for Credit Risk Management.": International Journal of Computer and Information Technology (ISSN: 2279 - 0764) Volume 03 Issue 06, November 2014 ) with another article awaiting publishing in the near future. Siqabukile has also presented a conference paper entitled "Wireless Security" at the ICT for Africa 2013 Conference held in Harare-Zimbabwe in February 2013. She is registered as a Technovation mentor to teach and motivate young

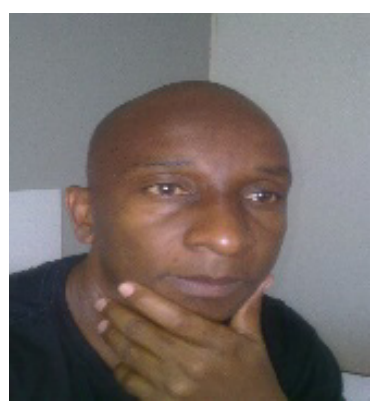

Thambo Nyathi is currently working towards his $\mathrm{PhD}$ with the University of Kwazulu Natal in South Africa. He holds a MSc in Computer Science and is an avid researcher interested in, Computer security, application of bio inspired techniques in cybersecurity.

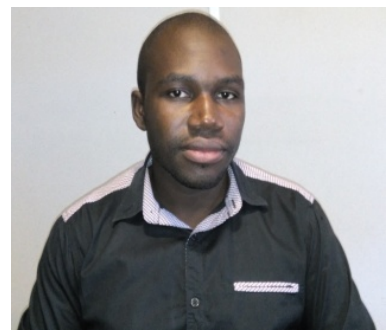

Khulekani Sibanda is a Lecturer in the department of computer science at the National University of science and technology. He holds a Master's Degree in Computer Science. His research interests lie in the fields of security, networking and business intelligence. Mr Sibanda's research has been published in IEEE sponsored conferences such as the IST Africa 